\title{
Representações sociais da velhice LGBT entre Agentes Comunitários de Saúde
}

\author{
Ludgleydson Fernandes de Araújo ${ }^{1}$ \\ Ana Gabriela Aguiar Trévia Salgado ${ }^{2}$ \\ José Victor De Oliveira Santos ${ }^{3}$ \\ Lorena Alves de Jesus ${ }^{4}$ \\ Luciana Kelly da Silva Fonseca ${ }^{5}$ \\ ${ }^{1}$ Universidade Federal do Piauí, PI, Brasil \\ https://orcid.org/0000-0003-4486-7565 \\ ${ }^{2}$ Universidade Federal do Piauí, PI, Brasil \\ https://orcid.org/0000-0001-7235-2599 \\ ${ }^{3}$ Universidade Federal do Piauí, PI, Brasil \\ https://orcid.org/0000-0002-6661-2873 \\ ${ }^{4}$ Universidade Federal do Piauí, PI, Brasil \\ https://orcid.org/0000-0001-7629-9676 \\ ${ }^{5}$ Universidade Federal do Piauí, PI, Brasil \\ https://orcid.org/0000-0001-8832-5261
}

\section{Resumo}

A pesquisa objetivou identificar as representações sociais da velhice LGBT entre Agentes Comunitários de Saúde. Participaram 50 profissionais, com idades entre 25 e 49 anos, de dois estados diferentes da Região Nordeste do Brasil. Foram utilizadas entrevistas semiestruturadas e questionários sociodemográficos analisados pelo software Iramuteq que classificou as representações sociais dentro de classes com proximidade lexical. A Classificação Hierárquica Descendente gerou seis classes a partir do corpus. Os resultados indicam representações que veem o idoso LGBT como ser de luta dentro do cenário excludente que há na sociedade. Ademais, as suas crenças geraram discursos que colocam a sua prática como potencial gestor de saúde ao segmento. Acredita-se que os dados registrados ofereçam base à discussão de vulnerabilidades e potencialidades da população pesquisada.

Palavras-chave: velhice, representação social, sexualidade, profissionais da saúde.

\section{Social Representations of LGBT old age among Community Health Agents}

\begin{abstract}
The research aimed to identify the social representations of LGBT old age among Community Health Agents. Fifty professionals, aged 25 to 49 years old, participated in two different states in the northeastern region of Brazil. Semi-structured interviews and sociodemographic questionnaires analyzed by Iramuteq software were used, which classified the social representations within classes with lexical proximity. The Descending Hierarchical Classification generated six classes from the corpus. The results indicate representations that see the elderly LGBT as being of struggle within the exclusionary scenario that exists in society. In addition, his beliefs generated speeches that put his practice as a potential health manager for the segment. It is believed that the recorded data provide a basis for the discussion of vulnerabilities and potentialities of the population surveyed.
\end{abstract}

Keywords: old age, social representation, sexuality, health professionals. 


\section{Representaciones Sociales de la Vejez LGBT Entre Agentes Comunitarios de Salud}

\section{Resumen}

La investigación objetivó identificar las representaciones sociales de la vejez LGBT entre Agentes Comunitarios de Salud. Participaron 50 profesionales, con edades entre 25 y 49 años, de dos estados diferentes de la región nordeste de Brasil. Se utilizaron entrevistas semiestructuradas y cuestionarios sociodemográficos analizados por el software Iramuteq que clasificó las representaciones sociales dentro de clases con proximidad lexical. La clasificación jerárquica descendiente generó seis clases a partir del corpus. Los resultados indican representaciones que ven el anciano LGBT como ser de lucha dentro del escenario excluyente que hay en la sociedad. Además, sus creencias generaron discursos que ponen su práctica como potencial gestor de salud al segmento. Se cree que los datos registrados se basan en la discusión de vulnerabilidades y potencialidades de la población investigada.

Palabras clave: vejez, representación social, sexualidad, profesionales de la salud.

O processo de envelhecimento corresponde à fase de desenvolvimento humano em que uma série de transformações biopsicossociais ocorre. O constante senso de adaptação imposto pelas condições advindas do período exige que novas formas de enfrentamento surjam (Santos et al., 2016). O envelhecimento pressupõe o surgimento de alterações físicas, psicológicas e sociais no indivíduo, sendo necessário considerá-las como naturais e gradativas. Além disso, essas transformações são gerais, podendo se manifestar em idade mais precoce ou mais avançada e em maior ou menor grau, de acordo com as idiossincrasias genéticas de cada indivíduo e principalmente com o modo de vida de cada um pelo caráter cumulativo (Zimerman, 2009).

É considerada uma experiência heterogênea, já que transcorre levando em consideração as diferentes nuances que norteiam o curso de vida dos indivíduos. Essas diferenças são determinadas por circunstâncias culturais e sociais, assim como fatores ligados à personalidade, à intelectualidade e também ao contexto de apoio que o indivíduo idoso se encontra (Neri, 2008). Pelo seu caráter plural, a velhice pode ser analisada por várias perspectivas, como a biológica, social, econômica, funcional e cronológica (Maia, 2011).

Os indicadores apontam para o fato de que o envelhecimento da população mundial alcança limites antes não possíveis, colocando a longevidade humana como um dos principais desafios para as instâncias sociais existentes (Instituto Brasileiro de Geografia e Estatística [IBGE], 2000, 2014, 2015). Nas últimas décadas o crescimento da população idosa passou a ser um fenômeno cada vez mais nítido. O crescimento vertiginoso do número de idosos no Brasil, até recentemente considerado um país jovem, começa a sinalizar para uma realidade diferente, trazendo a consciência de que a velhice existe e é uma questão social que exige uma atenção enorme (Zimerman, 2009). O Instituto Brasileiro de Geografia e Estatística (IBGE) confirma a tendência acelerada da população idosa no Brasil. Segundo o órgão, a população com essa faixa etária deve passar de 14,9 milhões (7,4\% do total), em 2013, para o número de 58,4 milhões (26,7\% do total) em 2060 , indicando ainda que nesse período a expectativa média de vida do idoso brasileiro deve aumentar dos atuais 75 anos para 81 anos de idade (IBGE, 2015).

Embora uma parcela significativa da população mundial ainda não tenha acesso à alimentação adequada e às condições de higiene essenciais, com os aprimoramentos da ciência e o uso de suas tecnologias, sobretudo no campo da medicina, se tornou possível um aumento cada vez maior da sobrevivência tanto de crianças como de adultos, motivada pela existência de investimentos em prevenção, promoção e educação em saúde, o surgimento de vacinas, medicamentos mais eficientes e cura para diversas doenças (Zimerman, 2009). Esses fatores ocasionaram mudanças significativas nos padrões de natalidade e mortalidade da população, gerando também uma mudança no perfil sociodemográfico de diversos países, incluindo o Brasil. O que era no passado privilégio de uma pequena parcela da população passou a ser uma 
experiência acessível a um número crescente de pessoas em todo o mundo. Por todos os progressos conquistados, envelhecer na atualidade já não é um benefício reservado à minoria populacional, conforme verificado nos últimos levantamentos do IBGE (2000, 2010, 2015).

Dentro do contexto social, é visível a influência restritiva de natureza excludente que a sociedade exerce sobre os idosos, a limitação de suas possibilidades de atuação, a opressão presente nas definições e padrões do "velho" que geram a impossibilidade de sua participação continuada em eixos importantes (Schneider \& Irigaray, 2008). Para Beauvoir (1990), essa atitude espontânea de recusar a velhice centra-se no fato da dificuldade de compreender que os velhos são semelhantes a qualquer indivíduo, uma vez que ela é definida por adjetivos que a tornam impotente. De maneira geral, não há uma velhice típica, considerada como padrão, universal e igual. O que há na realidade são múltiplas velhices, tantas como existem modelos de sociedades, culturas e classes sociais (Araújo \& Guarauto, 2010).

Uma série de estudos indica que a população idosa sofre marginalização, partindo da ótica social que analisa os indivíduos pelo desempenho do seu papel. Isso ocorre pela tendência da sociedade que prima pela produção de bens de consumo dentro da lógica capitalista como forma de indicar a sua inserção na organização social (Mendes, Gusmão, Faro, \& Leite, 2005). Em países em desenvolvimento, como o Brasil, as parcelas da população que não possuem condições de manejar a sua capacidade de trabalho passam a ser excluídas do processo produtivo que, no geral, indica utilidade social (Areosa, 2015).

Assim, é perceptível a necessidade dessa população ser assistida de forma adequada pelas políticas públicas de inclusão, como as ações previstas no Plano Nacional de Promoção da Cidadania e Direitos Humanos de LGBT (Brasil, 2009) e na Política Nacional de Saúde Integral de Lésbicas, Gays, Bissexuais, Travestis e Transexuais (Brasil, 2013) a fim de proporcionar espaços para a manifestação de suas potencialidades, possibilitando a criação de mecanismos que os auxi- liem a manter a sua idiossincrasia frente às ondas de preconceito encontrados nos mais variados espaços sociais além de permitir a sua adaptação.

Ser incluído dentro dos parâmetros etários e também sociais do que se considera velho, gera uma série de estereótipos que permeiam o imaginário social. Essas crenças situam-se, sobretudo, na consideração dessa fase como sendo de perdas em que as mudanças acarretam consequências que excluem práticas comuns em outras fases da vida. Na cultura ocidental, atualmente, há uma tendência a enquadrar a velhice com sinônimos que indicam além da decadência, perdas sociais e também biológicas (Groisman, 2002). Em contrapartida a essa concepção, alguns autores indicam a velhice como uma fase que possui tanto aspectos negativos - no que diz respeito às perdas relacionadas às capacidades físicas, sensoriais e neurológicas - quanto positivos - no que concerne às experiências adquiridas ao longo do longo caminho de vivências da pessoa idosa (Neri, 2008; Novaes, 1995).

O aumento da longevidade acarretou a necessidade de manejo adequado dessa faixa etária da população que socialmente encontra barreiras de natureza preconceituosa, refletida principalmente na desvalorização de potencialidades e no foco na crença errônea de que a velhice é uma fase de declínios em que práticas comuns em outros períodos são extintas, dentre os principais estigmas encontra-se o sexual (Araújo \& Carvalho, 2005; Debert, 2004; Silva, 2015). A vida sexual de uma pessoa não se extingue em idade avançada, o desejo e a afeição dirigidos ao outro não deixam de existir em nenhuma idade. Por ser considerada uma atividade pluralista, em que uma série de tendências e atividades podem ser encontradas, o que há na realidade é uma mudança da simbologia da sexualidade na velhice, visto que ela surge logo após o nascimento e finda praticamente na morte (Coelho, 2006).

Está em progresso na sociedade uma expansão constante para a manifestação das diferenças de todas as naturezas, incluindo a sexual. A diversidade está sendo aceita e a sua presença cada vez maior no cenário 
social, vem ganhando destaque pelas conquistas democráticas, tais como o de políticas e de direitos, mesmo que ainda haja a presença de um olhar estigmatizado e preconceituoso face às referidas demandas (Aragusuku \& Lopes, 2016). Atualmente, o movimento LGBT vem se tornando presente no contexto parlamentar, estando em pauta nas agendas junto ao governo e dialogando com lideranças políticas ganhando visibilidade $\mathrm{e}$ se constituindo como sujeitos que possuem direitos (Fernandez, 2010). A busca pela garantia de equidade vem se legitimando sobre a quebra da neutralidade dos agentes sociais responsáveis pela garantia de uma vida digna, através de projetos de leis - como o Projeto de Lei, n. ${ }^{\circ} 860$, de 2019-e de programas e ações instituídos pelos governos Federal e estaduais, além de iniciativas como o Seminário LGBT do Congresso Nacional.

Isso representa um grande avanço quando se considera a limitação das práticas das comunidades LGBT há algum tempo, onde a normativa que imperava baseava-se no modelo que tornava invisíveis as pessoas que se encontravam nesse contexto (Facchini \& França, 2009). Além disso, a carga de preconceito direcionada para essa parcela da população tinha natureza extrema pela negação de direitos, convertendo-se, como resultado, na ausência de instituições políticas e sociais que representassem proteção e garantia de cidadania (Marques \& Sousa, 2016).

Desde 2004, o Governo Federal brasileiro instituiu o "Brasil sem Homofobia - Programa de Combate à Violência e à Discriminação contra GLBT e de Promoção da Cidadania Homossexual", elaborado pela Secretaria Especial de Direitos Humanos (SEDH). O objetivo desse programa é incentivar a elaboração de políticas e ações contra a discriminação e que promovam o acesso igualitários aos serviços públicos para essas pessoas. Ainda em 2004, o Ministério da Saúde constituiu o Comitê Técnico de Saúde da População GLBT. Entre outras políticas implementadas pelo Governo, em 2013 foi criada a Política Nacional de Saúde Integral de Lésbicas, Gays, Bissexuais, Travestis e Transexuais. Nesse cenário, torna-se necessário garantir o acesso a essas políticas, explicitar direitos e encaminhar para uma autonomia cada vez maior as pessoas LGBT (Araújo \& FernándezRouco, 2016; Santos, Carlos, Araújo, \& Negreiros, 2017).

Apesar dessa consideração da abertura para novos comportamentos, ainda pesam as consequências do preconceito vigente. Há indícios de que os indivíduos idosos diretamente ligados a práticas homoafetivas estão de certa forma marcados pelo silêncio que é legitimado pelo estigma duplo da idade avançada e da sexualidade não normativa, além dos estigmas vivenciados e internalizados, que nutrem a exclusão social, a vitimização e, muitas vezes, a ocultação da sua identidade como uma consequência desse processo e como causa da diminuição da saúde entre os idosos LGBT (Fredriksen-Goldsen, 2016; Vries, 2015; Mota, 2009), sendo observável, por esse motivo, que a identidade de gênero e a orientação sexual de um idoso LGBT gera consequências em seu eixo psicológico e físico (Kimmel, 2015). Nesse sentido, o aumento da participação da população idosa homoafetiva nos movimentos e expressões ligadas às pessoas LGBT sugere que a presença de interação geracional soma força na busca pelos ideais de autonomia, socialização e igualdade legítima (IBGE, 2010; Côrrea-Ribeiro, Abdo, \& Camargos, 2016).

Nesse contexto de importância da atuação e das estratégias utilizadas pelos serviços de saúde, visto a sua capacidade de gerar mudanças sociais a partir da promoção de saúde, cabe ressaltar a atenção primária prestada pelas unidades básicas de saúde que tem como princípio atuar dentro do território dos usuários. $\mathrm{O}$ Programa Saúde da Família (PSF) objetiva, inicialmente, funcionar como gestor de ações voltadas para segmentos da população que possuem dificuldades de acesso aos serviços e vem se legitimando, em muitas regiões, como meio para ampliar o modelo de atenção (Silva, 2010).

Nessa perspectiva, vale destacar a atuação do Agente Comunitário de Saúde (ACS) na atenção básica. Esse profissional se estabelece como interlocutor das demandas que precisam ser comunicadas às unidades de saúde. O estudo de Silva e Dalmaso (2002) evidenciou que as atividades desenvolvidas pelos ACS se baseiam 
na atuação sobre situações da realidade da comunidade, buscando integrar essas informações aos demais profissionais da equipe de saúde, realizando assim a identificação de uma série de questões que podem ser alvo de intervenções. O seu papel, portanto, é o de funcionar como mediador que constrói o elo entre usuários e os serviços médicos (Filgueiras \& Silva, 2011).

O ACS, enquanto profissional que adentra livremente na comunidade, tem como função a identificação de necessidades de grupos específicos, devendo oferecer acolhimento dos segmentos que têm demandas de saúde clarificadas pelo nível de prejuízos em sua autonomia (Silva \& Dalmaso, 2002). Vale salientar que as ações interventivas de promoção à saúde, prevenção e recuperação só poderão ser efetivadas se ocorrer a comunicação fluida entre populações, grupos sociais, ACS e a equipe de saúde responsável pelo território (Brasil, 2012).

A Teoria das Representações Sociais é capaz de traduzir o conhecimento que norteia a prática e os discursos no cotidiano baseado no conhecimento adquirido pelo senso comum e compartilhado pela sua natureza coletiva, sendo essas concepções influenciadas pela bagagem cultural, os códigos, símbolos, valores, ideologias e tudo mais que faz parte do contexto em que se insere, auxiliando a construção da nossa realidade (Jodelet, 1989; Morera et al., 2015; Moscovici, 2003). Utilizar a Teoria das Representações Sociais se mostra útil pela sua capacidade de oferecer um panorama rico que incorpora o conhecimento individual ao que é compartilhado por um determinado grupo, permitindo conhecer quais os pressupostos em comum acerca da representação social (Doise, 1990).

Sob essa perspectiva, apreender a natureza da elaboração social que esses profissionais têm sobre o segmento LGBT e obter as representações sociais entre esses profissionais - essenciais na promoção de saúde - permite que seja alcançado subsídio para reflexões acerca de discursos e práticas. Visto isso, a presente investigação tem como objetivo principal identificar as representações sociais da velhice LGBT entre Agentes Comunitários de Saúde.

\section{Método}

Trata-se de uma pesquisa qualitativa, caracterizada por um método exploratório, com amostra não probabilística e por conveniência.

\section{Participantes}

Contou-se com 50 Agentes Comunitários de Saúde de ambos os sexos, com idades entre 25 e 49 anos de dois estados do Nordeste brasileiro. As mulheres compuseram a maior parte dos participantes do estudo (84\%). Entre os participantes, no que diz respeito ao estado civil, esses se declararam: $20 \%$ solteiros, $54 \%$ casados, $10 \%$ em união civil, $8 \%$ em relacionamento sério, $4 \%$ separados e $4 \%$ viúvos. No que diz respeito à escolaridade, $70 \%$ concluíram o Ensino Médio, 18\% concluíram o Ensino Fundamental, $8 \%$ possuem superior incompleto, totalizaram $8 \%$ da amostra o número de participantes com superior completo e pós-graduação. Quanto à orientação sexual, $88 \%$ dos participantes são heterossexuais e 12\% são homossexuais ou bissexuais. Em relação à religião, $68 \%$ são católicos, $10 \%$ protestantes, $8 \%$ de católicos não praticantes, $5 \%$ espíritas, os demais se identificam como ateus, agnósticos ou praticantes da umbanda. Em relação a ter recebido preparação com disciplina voltada à saúde do idoso, $88 \%$ declararam sim, $62 \%$ afirmaram ter parentesco com alguém de orientação homossexual e $48 \%$ da amostra declarou ter contato com idoso LGBT.

\section{Instrumentos}

Utilizou-se uma entrevista semiestruturada com a seguinte pergunta norteadora: "Como você entende a velhice LGBT?”. O instrumento inclui dados sociodemográficos para caracterização da amostra, contendo perguntas referentes a sexo, idade, estado em que vive, estado civil, renda mensal, religiosidade/espiritualidade, orientação sexual, escolaridade e tempo de atuação na área.

\section{Procedimentos éticos}

A presente pesquisa foi submetida ao Conselho de Ética em Pesquisa - CEP da Universidade Federal 
do Piauí, apresentando CAEE: 57225916.1.0000.5214 e número do parecer: 1.755.790. Os dados foram coletados em campo, buscando agentes comunitários em pleno exercício de suas atividades nos territórios sob serviço de Unidades Básicas de Saúde. A obtenção de participantes para compor a amostra se deu através da solicitação direta em preencher o instrumento da pesquisa. Durante a coleta dos dados, informações acerca dos objetivos do estudo, além do esclarecimento sobre a participação voluntária, anonimato e informações sobre o preenchimento do Termo de Consentimento Livre e Esclarecido, foram transmitidas. É pertinente salientar sobre a possibilidade de desistência a qualquer momento, que foi esclarecida ao participante, sendo de 20 minutos a média para preenchimento do questionário.

\section{Procedimentos de análises de dados}

Os dados sociodemográficos foram analisados pelo software SPSS for Windows versão 21. As entrevistas foram analisadas pelo software Iramuteq - Interface de R Pour Analyses Multidimensionnelles de Textes et de Questionnaires (Nascimento \& Menandro, 2006; Camargo \& Justo, 2013), responsável por analisar o corpus referente à pergunta respondida pelos componentes da amostra. Tal corpus foi estruturado no editor de texto OpenOfficeWriter, composto por 52 Unidades de Contextos Iniciais (UCI). As análises foram submetidas ao método da Classificação Hierárquica Descendente (CHD).

\section{Resultados}

O total de 50 entrevistas, ou unidades de contexto inicial (u.c.i), a partir da análise no software Iramuteq, resultou em 39 segmentos de texto classificados em 54 unidades de contexto elementar (u.c.e.), dando origem ao dendrograma (Figura 1). A análise identificou 4 classes de aproximação lexical e não foi possível determinar um perfil sociodemográfico dos participantes em cada uma delas. A primeira partição dividiu o corpus em dois subcorpus, separando a classe 3 das demais. A segunda partição fragmentou o subcorpus maior, originando a classe 4. A última partição deu origem às classes 1 e 2 .

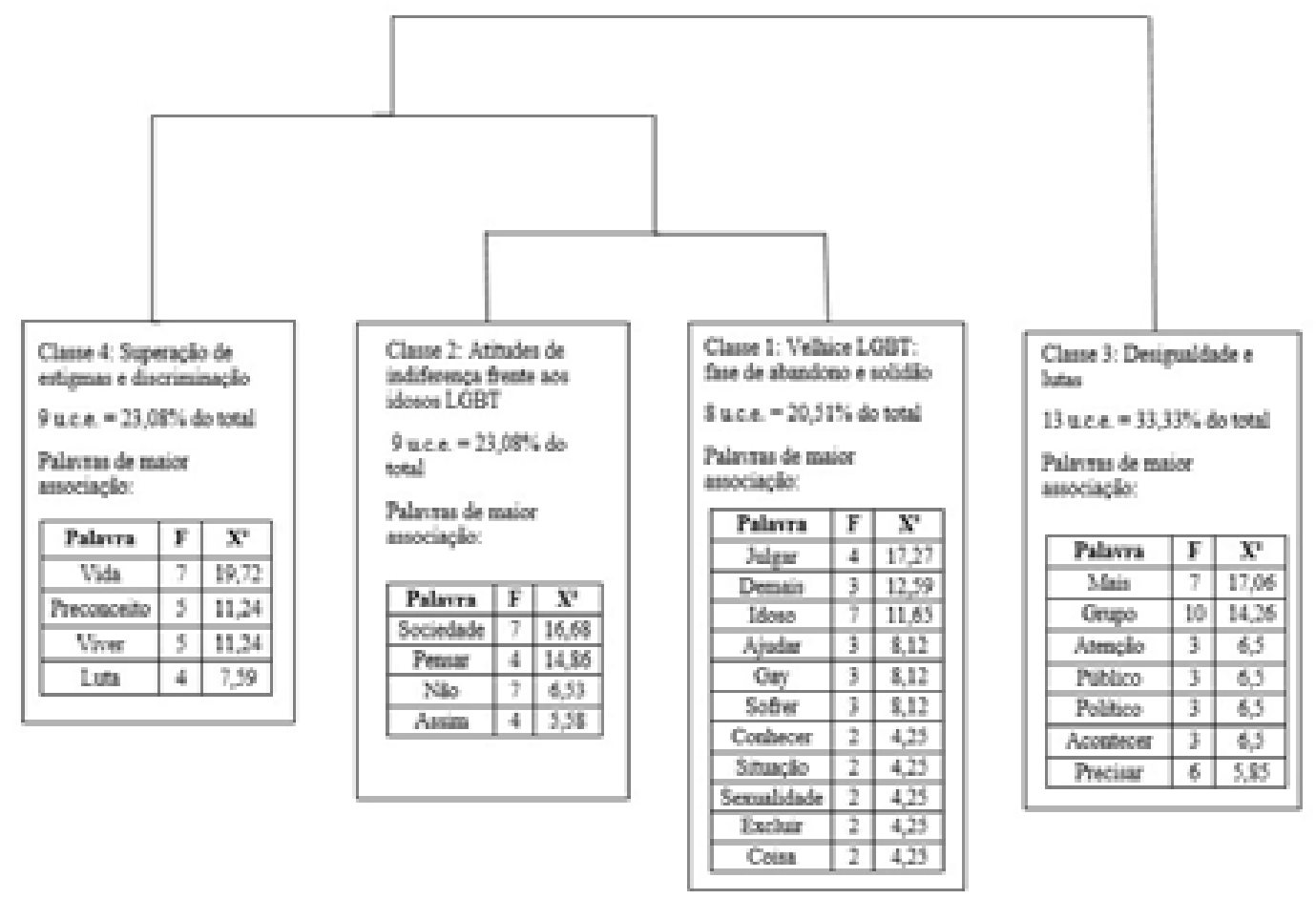

Figura 1 - Distribuição das classes nas representações sociais da velhice LGBT 
A classe 1, denominada Velhice LGBT: fase de abandono e solidão, é composta por 8 u.c.e., equivalente a $20,51 \%$ do total. No campo das representações sociais, os vocábulos se referem ao preconceito social diante do público LGBT, visto que são tidos como excluídos pela família e sociedade. As falas também demonstraram a importância do ACS no auxílio enquanto profissionais, no que se refere ao acesso aos serviços de saúde mediante as visitas domiciliares que proporcionam maior em relação às questões de saúde e apoio social.

Denominada Atitudes de indiferença frente aos idosos $L G B T$, a classe 2 é formada por 9 u.c.e., compondo $23,08 \%$ das unidades. Essa classe se aproxima da classe 1 em virtude dos discursos que representam a velhice como fase de exclusão social. Além disso, os conteúdos semânticos identificados na classe 2 remetem à invisibilidade dos idosos LGBT e ao preconceito que essa parcela da população é alvo.

A terceira classe tem $33,33 \%$ do total de u.c.e. (13 unidades), a maior classe. Os conteúdos semânticos dessa classe - denominada Desigualdade e lutas - faz referência ao preconceito, às dificuldades, à desigualdade e às lutas que permeiam a vida das pessoas LGBT e, portanto, dos idosos LGBT. Ficam enfatizadas também as necessidades no que diz respeito ao cuidado e ao acesso à saúde, que se configuram como uma realidade a ser ainda superada, principalmente, através de mais ações e políticas públicas.

Por último, a classe 4, denominada Superação de estigmas e discriminação, com $23,08 \%$ do total ( 9 unidades), é constituída de conteúdos semânticos que remetem ao reconhecimento e a cidadania conquistados ao longo da história de vida dos atuais idosos LGBT, marcada pela luta pelo fim do preconceito.

\section{Discussão}

Os dados obtidos nesta pesquisa após análise e interpretação demonstraram, de maneira geral, a presença de alguns estereótipos relacionados à velhice LGBT, além de uma visão de que essa fase da vida pode ser vivida de forma positiva pelas pessoas LGBT. Os conteúdos lexicais de cada classe identificada a partir do dendrograma serão discutidos.

\section{Classe 1 - Velhice LGBT: fase de abandono e solidão}

Os resultados obtidos na classe 1 relacionam a velhice LGBT ao preconceito e à exclusão social tida como comum nessa fase da vida. Contudo, reconhece-se que o idoso LGBT sofre duplamente com o estigma da idade e por não seguir a orientação heteronormativa. Essa concepção está compreensível nos seguintes discursos: "acredito que alguns sofrem pela solidão, os que eu conheço vivem sozinhos e precisam ser suficientes para si" (Agente 6, informação verbal, 13 de dezembro, 2017), "no meu território conheço idosos gays que se isolam e que foram excluidos por parentes e isso é triste demais, por isso tento ajudar no que posso quando faço visitas domiciliares" (Agente 29, informação verbal, 20 de dezembro, 2017) e "os casos que acompanhei são de idosos que são solitários e que a família excluiu muito cedo por isso alguns sofrem demais por essa situação e não encontram muito apoio por causa da sua sexualidade diferente dos outros" (Agente 49, informação verbal, 19 de janeiro, 2018).

O estereótipo da velhice como uma fase de abandono e solidão referida nos conteúdos dos participantes corrobora com estudos anteriores sobre as representações sociais da velhice (Camargo et al., 2014; Cruz \& Ferreira, 2011; Daniel, Antunes, \& Amaral, 2015).

A falta de apoio social - gerada, muitas vezes, pelo abandono da família em virtude da orientação sexual do indivíduo e a ausência de filhos e de um relacionamento duradouro - contribui para que as condições de saúde das pessoas idosas LGBT sejam ainda mais insatisfatórias, principalmente no que diz respeito à saúde mental desses sujeitos. A solidão e a ausência de apoio social causam sofrimento psíquico nesses idosos, aumentando o risco de serem desenvolvidos transtornos mentais, como a depressão (Carneiro, Falcone, Clark, Del Prette, \& Del Prette, 2007; Fredriksen-Goldsen et 
al., 2015; Guedea et al., 2006), além do maior uso de álcool e tabaco (Fredriksen-Goldsen et al., 2015).

\section{Classe 2 - Atitudes de indiferença frente aos idosos LGBT}

Essa classe apresenta, além das falas relacionadas à exclusão, conteúdos que fazem referência ao pouco conhecimento da sociedade sobre o grupo dos idosos LGBT, além de relatar o preconceito que existe para com essas pessoas. Os discursos mais representativos dessa classe são: “idosos que são assim sofrem muito preconceito e a gente quase não ouve falar deles na televisão, eu mesmo ainda não tinha parado pra pensar sobre eles" (Agente 51, informação verbal, 22 de janeiro, 2018), "entendo que são pessoas excluidas da sociedade e principalmente da família" (Agente 2, informação verbal, 05 de dezembro, 2017), "penso que sejam pessoas muito batalhadoras que viveram muitas coisas ruins por causa da sociedade que não aceita sua escolha de ser" (Agente 38, informação verbal, 29 de dezembro, 2017), "acho que as pessoas que fazem parte desse grupo são fortes porque a sociedade é muito preconceituosa e violenta com pessoas que elas não concordam" (Agente 41, informação verbal, 02 de janeiro 2018) e "penso que seja um grupo excluído da sociedade, pouco conheço sobre, não ouço falar ou vejo" (Agente 18, informação verbal, 16 de dezembro, 2017).

Os dados obtidos nessa classe confirmam o resultado obtido pelo estudo de Salgado et al. (2017) sobre a invisibilidade da população idosa LGBT. Esses autores justificaram essa invisibilidade pelo estereótipo de julgar os idosos como seres sem sexualidade, pela convicção de que todos os idosos são heterossexuais, pela dificuldade de acesso à essa população que sofre com o medo de ser vítima de preconceito e de perseguição e pelo desinteresse, por muitos anos, dos campos da Gerontologia e da Geriatria em estudar esse aspecto da vida dos idosos (Orel, 2014; Salgado et al., 2017).

Além disso, as concepções de atitudes preconceituosas em relação aos idosos LGBT também corroboram com os dados apresentados por Salgado et al. (2017), onde os participantes revelaram a existência do preconceito no seio da sociedade, contudo não se identificam como parte dessa sociedade que excluiu e trata com desrespeito as pessoas LGBT.

\section{Classe 3 - Desigualdade e lutas}

A classe 3 se caracteriza por discursos que designam a velhice LGBT como uma fase marcada ainda pela exclusão e pela invisibilidade, apesar de ter se configurado como um grupo revolucionário que lutou em busca de respeito e reconhecimento e se mobilizou a fim de dissolver os estigmas que lhes eram atribuídos. Essa concepção é identificada em discursos como "[...] exclusão e invisibilidade, esse é um segmento marginalizado" (Agente 3, informação verbal, 05 de dezembro, 2017) e "um grupo que revolucionou, lutou em busca de reconhecimento e aceitação" (Agente 4, informação verbal, 06 de dezembro, 2017).

Desde as décadas de 1960 e 1970 - que marcam uma crescente visibilidade e radicalização do movimento LGBT (Papalia, 2013), caracterizado por um discurso de autoafirmação e liberação, a exemplo de grupos como Society of Individual Rights, organização homossexual de São Francisco (Ca, Estados Unidos) que, pouco a pouco, acabou tomando boa parte do espaço ocupado pela Mattachine Society -, a população LGBT tem conquistado, além de reconhecimento, respeito e liberdade, proteção e direitos, através de políticas públicas e projetos de leis. Como exemplo, tem-se a Resolução 175, de 14 de maio de 2013 do Conselho Nacional de Justiça (CNJ) do Brasil, que dispõe sobre a habilitação, celebração de casamento civil e de conversão de união estável em casamento, entre pessoas de mesmo sexo, o que corrobora alguns dos discursos identificados nessa classe.

Além disso, nessa classe evidencia-se que os ACS admitem que esse grupo precisa de mais respeito e mais atenção. Alguns discursos também indicam a ausência de ações e políticas voltadas a esse público. Isto é perceptível a partir das falas "um grupo que precisa de atenção por 
necessitar de mais inclusão, é necessário respeito pelas políticas públicas é é nosso papel construir mais igualdade" (Agente 10, informação verbal, 08 de dezembro, 2017), “um processo com mais dificuldades por causa da ausência de politicas públicas que queiram amparar essa população" (Agente 16, informação verbal, 15 de dezembro, 2017), “é importante fazer dessas pessoas alvo de iniciativas de ações de saúde e considerá-los tão importantes como os grupos de gestantes e crianças" (Agente 47, informação verbal, 17 de janeiro 2018).

Apesar das conquistas desse público, as desigualdades, principalmente no que tange ao cuidado com a saúde, ainda são acentuadas e as políticas de atenção à saúde dessas pessoas ainda são pouco conhecidas. Entende-se que por esse motivo os participantes relataram a ausência de ações direcionadas à população LGBT. Fredriksen-Goldsen et al. (2015) apontam que as disparidades entre a saúde dos idosos LGBT e dos idosos heterossexuais ainda são inquietantes, principalmente por desvantagens ambientais, econômicas e sociais, bem como pela escassez de estudos acerca da gerontologia LGBT (Fredriksen-Goldsen, 2016; Orel, 2014). O pico da epidemia do vírus HIV, entre 1987 e 1996, intensificou o sentimento antigay e a homofobia, principalmente, nos Estados Unidos, quando foi feita a associação entre a homossexualidade e a presença da doença. Essas experiências despertam nos idosos LGBT, que na época tinham idades entre 25 e 44 anos, o receio de sofrer discriminação na busca por serviços de saúde e assistência social (Cahill, 2015).

Além disso, os idosos LGBT, principalmente os transexuais, apresentam necessidades particulares e mais específicas (Fernández-Rouco, Sánchez, \& González, 2012) e, apesar da Política Nacional de Saúde Integral de LGBT, implementada pelo Ministério da Saúde no Sistema Único de Saúde, a população idosa LGBT ainda é tratada com invisibilidade, visto que o preconceito e a discriminação também estão enraizados nos profissionais desse serviço (Tarquino, Santos, Coutinho, Lima Cruz, \& Brasil, 2015).

\section{Classe 4 - Superação de estigmas e discriminação}

Nessa classe, a velhice LGBT é representada como uma etapa bem vivida, mesmo que a vivência dessas pessoas seja buscando enfrentar e superar o preconceito vigente na sociedade, um modo de viver pautado na luta pela conquista de direitos e no desejo de ser livre do preconceito e dos estigmas sociais. Algumas falas simbolizam essas concepções, por exemplo, “[...] como um modo de vida de luta e busca por viver saudavelmente vencendo as opiniões de quem não aceita a sua vida" (Agente 7, informação verbal, 13 de dezembro, 2017), "[...] tem muita luta para se sentir inserido e usar dos serviços" (Agente 40, informação verbal, 02 de janeiro, 2018), "é sinônimo de luta e muita resistência para enfrentar o preconceito que as pessoas têm por causa do seu estilo de vida que não se encaixa nos padrões" (Agente 24, informação verbal, 18 de dezembro, 2017) e "um grupo heterogêneo em que existe muito amor e o desejo de ser livre do preconceito vivido no dia a dia" (Agente 9, informação verbal, 08 de dezembro, 2017).

Verificou-se nessa classe falas que remetem à ideia de velhice LGBT como sinônimo de luta, superação e resistência. De modo geral, o público LGBT enfrenta uma sequência de dificuldades ao longo de sua biografia-violências motivadas pelos estereótipos negativos, discriminação e intolerância, discriminação, estigmas e dificuldade de aceitação (Salgado et al., 2017) - e as pessoas idosas LGBT encaram esses mesmos obstáculos e, ainda, o estigma da idade.

Contudo, os direitos e reconhecimento alcançados durante a trajetória de vida dessas pessoas, como o direito à vida, à dignidade, à igualdade, à liberdade sexual e de envelhecer com segurança, faz com que a velhice seja interpretada por esse grupo como uma fase de vitória e de orgulho (Henning, 2013), confirmando o entendimento dos participantes dessa pesquisa. 


\section{Considerações finais}

Buscar compreender o significado das representações sociais de profissionais da saúde, como os Agentes Comunitários de Saúde, é essencial quando se pensa nesses profissionais como promotores de saúde e de qualidade de vida, principalmente na população idosa. Os valores que atravessam a relação que entre as práticas desses profissionais e os discursos voltados para o segmento LGBT envelhecido permitem a visualização de como esse público está sendo assistido pelas políticas públicas gestoras de saúde e de que forma essas políticas têm sido eficazes para que se promova um atendimento baseado na equidade.

É pertinente inferir sobre a capacidade do estudo de abarcar a contextualização entre as crenças e os conhecimentos que consolidam as representações sociais dos ACS com o cenário atual que o público LGBT se depara ao buscar direitos e serviços. No geral, através da pesquisa foi possível constatar que a percepção dos ACS, enquanto profissionais comprometidos com o bem-estar da população que eles assistem, centra-se na crença da existência do preconceito direcionado a esse se grupo, o sofrimento e as vulnerabilidades advindas do processo de estigmatização. A empatia para com o idoso LGBT existe permanentemente dentro dos discursos registrados, a valorização do público ocorre como resultado da relevância dada ao seu poder de resignar o preconceito social e sobrepor a sua saúde ao processo doloroso vivenciado na sua trajetória de vida.

Espera-se que os resultados obtidos nessa pesquisa possam oferecer subsídios para construção de novas reflexões, problematizações e perspectivas que se encaminhem para uma assistência adequada à população LGBT.

\section{Referências}

Aragusuku, H. A., \& Lopes, M. (2016). Preconceito, Discriminação e Cidadania LGBT: Políticas Públicas em Mato Grosso e no Brasil. Revista de Antropologia do Centro-Oeste, 3(5), 17.
Araújo, L. F., \& Carvalho, V. Â. M. D. L. (2005). Aspectos sócio-históricos e psicológicos da velhice. Mneme - Revista de Humanidades, 6(13), 228-236.

Araújo, A. O., \& Guarato, D. Z. (2010). Envelhecimento e saúde mental: atividade física, envelhecimento e a manutenção da saúde. Uberlândia: Edufu. https://doi. org/10.14393/edufu-978-85-7078-254-0

Araújo, L. F., \& Fernández-Rouco, N. (2016). Idosos LGBT: Fatores de Risco e Proteção. Em Falcão, D. V. da S., Pedroso, J. da S., \& Araújo, L. F. de (orgs.). Velhices: temas emergentes nos contextos psicossocial e familiar (pp. 129-138). Campinas: Alínea.

Areosa, S. V. (2015). Envelhecimento e universidade: Um estudo do fórum gaúcho das IES do Rio Grande do Sul. São Leopoldo: Casa Leiria Editora. https://doi. org/10.17533/udea.iee.v33n1a18

Beauvoir, S. A. (1990). A velhice. Rio de Janeiro: Nova Fronteira.

Brasil. (2012). Política Nacional de Atenção Básica. Brasília: Ministério da Saúde.

Brasil . (2009). Plano Nacional de Promoção da Cidadania e Direitos Humanos de LGBT. Brasília: Secretaria Especial dos Direitos Humanos. https://doi. org/10.1590/1413-81232014198.06322014

Brasil . (2013). Política Nacional de Saúde Integral de Lésbicas, Gays, Bissexuais, Travestis e Transexuais (LGBT). Brasília: Ministério da Saúde. https://doi. org/10.1590/s0034-89102008000300027

Cahill, S. (2015). Community resources and government services for lgbt older adults and their families. In $\mathrm{N}$. A. Orel \& C. A. Fruhauf (Orgs.). The lives of LGBT older adults: Understanding challenges and resilience (pp. 141-169). Washington, DC: American Psychological Association. https://doi.org/10.1037/14436-007

Camargo, B. V., Contarello, A., Wachelke, J. F. R., Morais, D. X., \& Piccolo, C. (2014). Representações sociais do envelhecimento entre diferentes gerações no Brasil e na Itália. Psicologia em Pesquisa, 8(2), 179-188.

Camargo, B. V., \& Justo, A. M. (2013). IRAMUTEQ: um software gratuito para análise de dados textuais. Temas em Psicologia, 21(2), 513-518. https://doi.org/10.9788/ tp2013.2-16 
Carneiro, R. S., Falcone, E., Clark, C., Del Prette, Z., \& Del Prette, A. (2007). Qualidade de vida, apoio social e depressão em idosos: relação com habilidades sociais. Psicologia: Reflexão e Crítica, 20(2), 229-237. https:// doi.org/10.1590/s0102-79722007000200008

Coelho, A. V. R. (2006). O Sentido subjetivo da sexualidade na terceira idade. Monografia (Mestrado em Psicologia) - Universidade Católica de Goiás, Goiânia, Brasil.

Corrêa-Ribeiro, R., Abdo, C. H. N., \& E. F. Camargos. (2016). Lésbicas, gays e bissexuais idosos no contexto do envelhecimento. Geriatrics, Gerontology and Aging, 10(3), 158-163. https://doi.org/10.5327/z2447$\underline{211520161600023}$

Cruz, R. C., \& Ferreira, M. A. (2011). Um certo jeito de ser velho: representações sociais da velhice por familiares de idosos. Texto \& Contexto Enfermagem, 1, 144151. https://doi.org/10.1590/s0104-07072011000100017

Facchini, R., \& França, I. L. (2009). De cores e matizes: sujeitos, conexões e desafios no Movimento LGBT brasileiro. Sexualidad, Salud y SociedadRevista Latinoamericana, (3), 54-81. Recuperado de: http://www.e-publicacoes.uerj.br/index.php/ SexualidadSaludySociedad/article/view/41/466

Daniel, F., Antunes, A., \& Amaral, I. (2015). Representações sociais da velhice. Análise Psicológica, 33(3), 291-301. https://doi.org/10.14417/ap.972

Debert, G.G. (2004). A reinvenção da velhice: Socialização e Processos de Reprivatização do Envelhecimento. São Paulo: Ed. Da Universidade de São Paulo, Fapesp. https://doi.org/10.11606/issn.23188235.v48i0p219-265

Doise, W. (1990). Les représentations sociales. Traité de Psychologie Cognitive, 3, 190-198.

Fernandez, O. (2010). Homossexuais, cidadania e direitos humanos no Brasil. In G. Venturi (Org.). Direitos humanos: percepções da opinião pública. Brasília-DF: Secretaria de direitos humanos da presidência da república. https://doi.org/10.26512/2014.11.d.18267

Fernández-Rouco, N., Sánchez, F. L., \& González, R. J. C. (2012). Transexualidad y vejez: una realidad por conocer. Kairós Gerontologia. Revista da Faculdade de Ciências Humanas e Saúde, 15(3), 15-25.
Filgueiras, A.S, \& Silva A. L. A. (2011). Agente Comunitário de Saúde: um novo ator no cenário da saúde do Brasil. Physis, 21 (3),899-916. https://doi. org/10.1590/s0103-73312011000300008

França, I. L. (2006). Cercas e pontes: o movimento GLBT e o mercado GLS na cidade de São Paulo. Dissertação (Mestrado em Antropologia Social) Faculdade de Filosofia, Letras e Ciências Humanas, Universidade de São Paulo, São Paulo. https://doi. org/10.11606/d.8.2006.tde-03092007-141155

Fredriksen-Goldsen, K. I. (2016). The Future of LGBT+ Aging: A Blueprint for Action in Services, Policies, and Research. Generations, 40(2), 6-15.

Fredriksen-Goldsen, K. I., Hoy-Ellis, C. P., Muraco A., Goldsen, J., \& Kim, H. J. (2015). The health and well-being of lgbt older adults: disparities, risks, and resilience across the life course. In N. A. Orel \& C. A. Fruhauf (Orgs.). The lives of LGBT older adults: Understanding challenges and resilience (pp. 25-53). Washington, DC: American Psychological Association. https://doi.org/10.1037/14436-002

Groisman, D. (2002). A velhice entre o normal e o patológico. História, Ciência, Saúde. 9, 61-78. https:// doi.org/10.1590/s0104-59702002000100004

Guedea, M. T. D., Albuquerque, F. J. B., Tróccoli, B. T., Noriega, J. A. V., Seabra, M. A. B., \& Guedea, R. L.D. (2006). Relação do bem-estar subjetivo, estratégias de enfrentamento e apoio social em idosos. Psicologia: Reflexão e Crítica, 19(2), 301-308. https:// doi.org/10.1590/s0102-79722006000200017

Henning, C. E. (2013, setembro). O panorama heteronormativo sobre a velhice e a literatura que entrelaça homossexualidade, bissexualidade, transgêneros e envelhecimento. Seminário Internacional Fazendo Gênero. Florianópolis, Santa Catarina, Brasil, 10.

Instituto Brasileiro de Estatística - IBGE (2000). Censo Demográfico 2000. Rio de Janeiro: IBGE.

Instituto Brasileiro de Estatística - IBGE . (2010). Censo Demográfico 2010. Rio de Janeiro: IBGE.

Instituto Brasileiro de Estatística - IBGE . (2014). Pesquisa nacional por amostra de domicílios. Rio de Janeiro: IBGE. https://doi.org/10.21710/rch.v25i0.451 
Instituto Brasileiro de Estatística - IBGE . (2015). Pesquisa nacional por amostra de domicílios. Rio de Janeiro: IBGE. https://doi.org/10.21710/rch.v25i0.451

Jodelet, D. (1989). Representações Sociais: fenômeno, conceito e teoria. Paris: Presses Universitares de France.

Kimmel, D. C. (2015). Theories of aging applied to LGBT older adults and their families. In N. A. Orel \& C. A. Fruhauf (Orgs.). The lives of LGBT older adults: Understanding challenges and resilience (pp. 73-90). Washington, DC: American Psychological Association. https://doi.org/10.1037/14436-004

Maia, F. O. M (2011). Vulnerabilidade e envelhecimento: panorama dos idosos residentes no município de São Paulo. (Tese de Doutorado em Enfermagem na Saúde do Adulto). Escola de Enfermagem, Universidade de São Paulo, São Paulo, Brasil. https://doi.org/10.11606/t.7.2011. $\underline{\text { tde-24102011-080913 }}$

Marques, F. D., \& Sousa, L. (2016). Portuguese Older Gay Men: Pathways to Family Integrity. Paidéia, 26(64), 149-159. https://doi.org/10.1590/1982-43272664201602

Mendes, M. R., Gusmão, J. L. D., Faro, A. C. M., \& Leite, R. D. C. B. D. (2005). A situação social do idoso no Brasil: uma breve consideração. Acta paulista de enfermagem, 18(4), 422-426. https://doi.org/10.1590/ s0103-21002005000400011

Morera, J. A. C., Padilha, M. I., Silva, D. G. V. da, \& Sapag, J. (2015). Aspectos teóricos e metodológicos das Representações Sociais. Texto \& Contexto - Enfermagem, 24(4), 1157-1165. https://doi. org/10.1590/0104-0707201500003440014

Moscovici, S. (2003). Representações Sociais: investigações em Psicologia Social. Petrópolis: Vozes.

Mota, M. P. (2009). Homossexualidade e Envelhecimento: algumas reflexões no campo da experiência. Revista Sinais, 1(06), 1981-3988.

Nascimento, A. R. A., \& Menandro, P. R. M. (2006). Análise lexical e análise de conteúdo: uma proposta de utilização conjugada. Estudos e Pesquisas em Psicologia, 6(2), 1-17.

Neri, A. L. (2008). Palavras-chave em gerontologia. 2a ed. Campinas: Alínea.
Novaes, M. H. (1995). Psicologia da terceira idade: conquistas possiveis e rupturas necessárias. Rio de Janeiro: Grypho.

Orel, N. A. (2014). Investigating the needs and concerns of lesbian, gay, bisexual, and transgender older adults: The use of qualitative and quantitative methodology. Journal of homosexuality, 61(1), 53-78. https://doi.org 110.1080/00918369.2013.835236

Papalia, D. E., \& Feldman, R. D. (2013). Desenvolvimento humano. Porto Alegre: Artmed.

Salgado, A. G. A. T., Araújo, L. F., Santos, J. V. O., Jesus, L. A, Fonseca, L. K. S., \& Sampaio, D. S. (2017). Velhice LGBT: uma análise das representações sociais entre idosos brasileiros. Ciencias Psicológicas, 11(2), 155-163. https://doi.org/10.22235/cp.v11i2.1487

Santos, J. V. O., Vasconcelos, M. S., Camelo, L. C. S. D., Silva, R. S. S. S., Morais, T. V., Araújo, L. F. (2016). Intervenção psicogerontológica num grupo de convivência para idosos: Um relato de experiência. Psicopedagogia On Line, 17, 31-41.

Santos, J. V. O., Carlos, K. P. T., Araújo, L. F., \& Negreiros, F. (2017). Compreendendo a velhice LGBT: uma revisão da literatura. In L. F. Araújo; C. M. R. G. Carvalho. (Org.). Envelhecimento e Práticas Gerontológicas, (1 ed., pp. 81-96). Curitiba-PR/TeresinaPI, Editora: CRV/EDUFPI.

Schneider, R. H., \& Irigaray, T. Q. (2008). O envelhecimento na atualidade: aspectos cronológicos, biológicos, psicológicos e sociais. Estud. psicol. (Campinas), 25(4), 585-593. https://doi.org/10.1590/ s0103-166x2008000400013

Silva, N. R. N. (2015). Sexualidade na velhice: a visão do idoso e os fatores influenciadores. Trabalho de Conclusão de Curso - (Bacharel em Terapia Ocupacional). Faculdade de Ceilândia, Universidade de Brasília, Brasília, DF, Brasil. https://doi.org/10.17013/risti.28.41-56

Silva, J. A.\& Dalmaso, A. S. W. (2002). Agente Comunitário de Saúde: o ser, o saber, o fazer. Rio de Janeiro: Fiocruz. https://doi.org/10.7476/9788575416136.0004 
Tarquino, M. L., Santos, L. V., Coutinho, M. I. B., Lima Cruz, L. H. de, \& Brasil, M. L. (2015, setembro). Invisibilidade na assistência: um enfoque na atenção à saúde da população LGBT idosa. Congresso Internacional de Envelhecimento Humano, Campina Grande, Paraíba, Brasil, 4.

Vries, B. (2008). Lesbian, gay, bisexual and transgender persons in later life. In D. Carr (Ed.), Encyclopedia of the life course and human development (pp. 161-165). Farmington Hills, MI: Gale.

Vries, B. (2015). Stigma and LGBT aging: Negative and positive marginality. In N. A. Orel \& C. A. Fruhauf (Orgs.). The lives of LGBT older adults: Understanding challenges and resilience (pp. 55-72). Washington, DC: American Psychological Association. https://doi. org/10.1037/14436-003

Zimerman, G. I. (2009). Velhice: Aspectos Biopsicossociais. São Paulo: Artmed.

Recebido em: 7/5/2018.

Aprovado em: 13/6/2019.

Publicado em: $\mathrm{xx} / \mathrm{x} / \mathrm{xxxx}$

Endereço para correspondência: Ludgleydson

Fernandes de Araújo (Universidade Federal do Piauí, Campus de Parnaíba, Departamento de Psicologia.

Av. São Sebastião, 2819, Cidade Universitária 64202020 - Parnaíba, PI - Brasil; (86)99990-9208/

Fax: (86)3323-5248; ludgleydson@yahoo.com.br)

\section{Nome: Ludgleydson Fernandes de Araújo.}

E-mail: ludgleydson@yahoo.com.br

Titulação Acadêmica: Doutorado em Psicologia, Universidade de Granada, UGR, Espanha.

Afiliação Institucional: Professor no quadro Permanente do Programa de Pós-Graduação em Psicologia (Stricto Sensu) do Campus Ministro Reis Velloso da Universidade Federal do Piauí (UFPI).

\section{Nome: Ana Gabriela Aguiar Trévia Salgado.}

E-mail: gabrielatrevia@outlook.com
Titulação Acadêmica: Mestranda em Psicologia pela Universidade Federal do Piauí.

Afiliação Institucional: Universidade Federal do Piauí, UFPI. Campus Ministro Reis Velloso.

\section{Nome: José Victor de Oliveira Santos.}

E-mail: victorolintos@hotmail.com

Titulação Acadêmica: Mestrando em Psicologia pela Universidade Federal do Piauí, UFPI.

Afiliação Institucional: Universidade Federal do Piauí, UFPI. Campus Ministro Reis Velloso.

\section{Nome: Lorena Alves de Jesus.}

E-mail: lorenaalve_s@hotmail.com

Titulação Acadêmica: Graduanda em Psicologia pela Universidade Federal do Piauí.

Afiliação Institucional: Universidade Federal do Piauí, UFPI. Campus Ministro Reis Velloso.

\section{Nome: Luciana Kelly da Silva Fonseca.}

E-mail: 1.kelly_fonseca@hotmail.com

Titulação Acadêmica: Graduada em Psicologia pela Universidade Federal do Piauí.

Afiliação Institucional: Universidade Federal do Piauí, UFPI. Campus Ministro Reis Velloso. 